\title{
Transversal Jacobi Operators in Almost Contact Manifolds
}

\author{
Jong Taek Cho ${ }^{1, *(\mathbb{D}}$ and Makoto Kimura ${ }^{2} \mathbb{0}$ \\ 1 Department of Mathematics, Chonnam National University, Gwangju 61186, Korea \\ 2 Department of Mathematics, Faculty of Science, Ibaraki University, Mito, Ibaraki 310-8512, Japan; \\ makoto.kimura.geometry@vc.ibaraki.ac.jp \\ * Correspondence: jtcho@chonnam.ac.kr
}

check for updates

Citation: Cho, J.T.; Kimura, M. Transversal Jacobi Operators in Almost Contact Manifolds. Mathematics 2021, 9, 31. https://dx.doi.org/10.3390/ math9010031

Received: 23 November 2020 Accepted: 21 December 2020 Published: 24 December 2020

Publisher's Note: MDPI stays neutral with regard to jurisdictional claims in published maps and institutional affiliations.

Copyright: () 2020 by the authors. Licensee MDPI, Basel, Switzerland. This article is an open access article distributed under the terms and conditions of the Creative Commons Attribution (CC BY) license (https: / / creativecommons.org/ licenses/by/4.0/).

\begin{abstract}
Along a transversal geodesic $\gamma$ whose tangent belongs to the contact distribution $D$, we define the transversal Jacobi operator $R_{\gamma}=R(\cdot, \dot{\gamma}) \dot{\gamma}$ on an almost contact Riemannian manifold $M$. Then, using the transversal Jacobi operator $R_{\gamma}$, we give a new characterization of the Sasakian sphere. In the second part, we characterize the complete ruled real hypersurfaces in complex hyperbolic space.
\end{abstract}

Keywords: almost contact manifold; transversal Jacobi operator; Sasakian sphere; ruled real hypersurface

MSC: 53C15; 53C25

\section{Introduction}

A smooth manifold $M^{2 n-1}$ is an almost contact manifold if its structure group of the linear frame bundle is reducible to $U(n-1) \times\{1\}$ [1]. Given an almost contact manifold, two associated structures enrich the geometry. One is a Riemannian metric $g$ compatible to a given almost contact structure and we obtain a almost contact Riemannian manifold $(M ; \eta, \xi, \phi, g)$. The other is an almost $C R$ structure $\mathcal{H}=\{X-i J X: X \in \Gamma(D)\}$, that is, each fiber $\mathcal{H}_{p}, p \in M$, is of complex dimension $n-1, \mathcal{H} \cap \overline{\mathcal{H}}=\{0\}$ and $[\mathcal{H}, \mathcal{H}] \subset \mathbb{C} D$, where $J=\left.\phi\right|_{D}$ and $D=$ Ker of $\eta$. In addition, the almost complex structure $J$ satisfies the integrability condition: $[\mathcal{H}, \mathcal{H}] \subset \mathcal{H}$, we call it an (integrable) $C R$ structure. S. Webster [2] introduced the terminology pseudo-Hermitian structure on real hypersurfaces in complex manifolds by such an $\eta$. The notion was also studied intensively and independently by $\mathrm{N}$. Tanaka [3].

On the other hand, in Riemannian geometry the Jacobi operator $R_{\gamma}=R(\cdot, \dot{\gamma}) \dot{\gamma}$ along geodesics $\gamma$ plays an important role, where $R$ denotes the Riemannian curvature tensor. In these circumstances, it is very interesting to study the behavior of Jacobi operators on almost contact Riemannian manifolds particularly in connection with their associated almost CR-structure.

This paper contains mainly two parts: in the first part, we give a characterization of the Sasakian sphere by using the transversal Jacobi operator $R_{\gamma}=R(\cdot, \dot{\gamma}) \dot{\gamma}$ along geodesics $\gamma$ whose tangent $\dot{\gamma}$ belongs to the contact distribution $D$. More precisely, we prove that a complete and simply connected $K$-contact manifold $M$ is isometric to the unit sphere if and only if the contact distribution $D$ is invariant by the transversal Jacobi operator $R_{\gamma}\left(R_{\gamma}(D) \subset D\right)$ and, at the same time, it is invariant by the covariant derivative $R_{\gamma}^{\prime}=\left(\nabla_{\dot{\gamma}} R\right)(\cdot, \dot{\gamma}) \dot{\gamma}\left(R_{\gamma}^{\prime}(D) \subset D\right)$ for any transversal geodesic $\gamma($ Corollary 1$)$. In the second part, we treat real hypersurfaces of a complex hyperbolic space, and then we prove a complete real hypersurface $M$ in a non-flat complex space form $\widetilde{M}_{n}(c), c \neq 0$ is congruent to a complete ruled real hypersurface in complex hyperbolic space $H_{n} \mathbb{C}$ if and only if the contact ditribution $D$ is invariant by the transversal Jacobi operator $R_{\gamma}$ and at the same time it is invariant by the covariant derivative $R_{\gamma}^{\prime}$ for any transversal geodesic $\gamma$ (Corollary 2). 


\section{Preliminaries}

First, we collect some basic notions and properties of almost contact Riemannian manifolds and strictly pseudo-convex pseudo-Hermitian manifolds.

Definition 1 ([1,4]). A (2n-1)-dimensional manifold $M$ is said to be an almost contact manifold if its structure group of the linear frame bundle is reducible to $U(n-1) \times\{1\}$, or equivalently, if there exist a $(1,1)$-tensor field $\phi$, a vector field $\xi$ and a 1-form $\eta$ satisfying

$$
\eta(\xi)=1 \text { and } \phi^{2}=-I+\eta \otimes \xi,
$$

where I denotes the identity transformation. We call $(\eta, \xi, \phi)$ an almost contact structure and the structure vector field $\xi$ the Reeb vector field in particular.

Then, there is a compatible Riemannian metric $g$ :

$$
g(\phi X, \phi Y)=g(X, Y)-\eta(X) \eta(Y)
$$

for any vector fields $X$ and $Y$ on $M$. Such a metric is called an associated metric and $(M ; \eta, \xi, \phi, g)$ (shortly, $M$ ) is said to be an almost contact Riemannian manifold.

For the exterior differential operator $d$, if $M$ satisfies in addition $d \eta=\Phi$, then $M$ is called a contact Riemannian manifold. Here, $\Phi(X, Y)=g(X, \phi Y)$ for any vector fields $X, Y$. On a contact Riemannian manifold, $\eta$ is a contact form, i.e., $\eta \wedge(d \eta)^{n-1} \neq 0$. Therefore, every contact Riemannian manifold is orientable. From (1) and (2) it follows that

$$
\phi \xi=0, \quad \eta \circ \phi=0, \quad \eta(\cdot)=g(\cdot, \xi) .
$$

Given a contact metric manifold $M$, we define the structure operator $h$ by $h=\frac{1}{2} \mathfrak{L}_{\xi} \phi$, where $\mathfrak{L}_{\xi}$ denotes the Lie derivative with respect to $\xi$. Then, we see that $h$ is self-adjoint and satisfies

$$
\begin{aligned}
& h \xi=0 \text { and } h \phi=-\phi h, \\
& \nabla_{X} \xi=-\phi X-\phi h X,
\end{aligned}
$$

where $\nabla$ is the Levi-Civita connection on $M$ associated with $g$. From (3) and (4), we see that $\xi$ is a geodesic vector field. Moreover, using (4) it is easy to see that $\xi$ is a Killing vector field if and only if $h$ vanishes. Such a contact Riemannian manifold is called a K-contact manifold.

Now, we recall the definition of CR structure.

Definition 2 ([5]). Let $M$ be a $(2 n-1)$-dimensional manifold and TM be its tangent bundle. $A C R$ structure on $M$ is a complex rank $(n-1)$ sub-bundle $\mathcal{H} \subset \mathbb{C} T M=T M \otimes \mathbb{C}$ satisfying (i) $\mathcal{H} \cap \overline{\mathcal{H}}=\{0\}$ and (ii) $[\mathcal{H}, \mathcal{H}] \subset \mathcal{H}$ (integrability), where $\overline{\mathcal{H}}$ denotes the complex conjugation of $\mathcal{H}$.

For a $\mathrm{CR}$ structure $\mathcal{H}$, we have a unique sub-bundle $D=\operatorname{Re}\{\mathcal{H} \oplus \overline{\mathcal{H}}\}$ and a unique bundle map $J$ such that $J^{2}=-I$ and $\mathcal{H}=\{X-i J X \mid X \in D\}$. If $M$ is orientable, then there exists a nowhere zero section $\eta$, i.e., a real one-form on $M$ such that $\operatorname{Ker}(\eta)=D$. For such $(\eta, J)$ the Levi form is defined by

$$
L(X, Y)=-d \eta(X, J Y)
$$

for $X, Y \in D$. If the Levi form is positive (or negative) definite and hermitian, then $(\eta, J)$ is called a strictly pseudo-convex pseudo-Hermitian CR structure. In general, when the Levi-form is non-degenerate there exists a unique globally defined nowhere zero tangent vector field $\xi$ such that $i_{\xi} d \eta=0$ and $\eta(\xi)=1$, where $i_{X}$ denotes the interior product with a vector field $X$ on $M$. Define the Webster metric (Definition 1.10 in [5]) on $M$ by

$$
g_{\eta}=L+\eta \otimes \eta,
$$


where $i_{\xi} L=0$.

Returning to an almost contact manifold $M=(M ; \eta, \xi, \phi)$, at each point $p \in M$ we denote $D_{p}=\left\{v \in T_{p} M \mid \eta(v)=0\right\}$. Then, $D: p \rightarrow D_{p}$ defines a distribution orthogonal to $\xi$, which is called a contact distribution. Then, the restriction $J=\left.\phi\right|_{D}$ of $\phi$ to $D$ defines an almost complex structure in $D$. Such $(\eta, J)$ is called an almost $C R$ structure, if $M$ satisfies

$$
[J X, J Y]-[X, Y] \in D(\text { or }[J X, Y]+[X, J Y] \in D)
$$

for all $X, Y \in D$. Furthermore, when it satisfies

$$
[J, J](X, Y)=0,
$$

where $[J, J]$ is the Nijenhuis torsion of $J$, the pair $(\eta, J)$ is a pseudo-Hermitian (integrable) $\mathrm{CR}$ structure associated with the almost contact structure $(\eta, \xi, \phi)$. On the other hand, if the Nijenhuis torsion of $\phi$ satisfies $[\phi, \phi]=-2 d \eta \otimes \xi$, then $M$ is said to be normal [6]. The associated pseudo-Hermitian structure of a normal almost contact structure is CR-integrable.

A contact Riemannian manifold with normality is called a Sasakian manifold. Therefore, every Sasakian manifold has an integrable CR-structure. Indeed, for a contact Riemannian manifold $(M ; \eta, \xi, \phi, g)$, CR-integrability is equivalent to the condition $\Omega=0$, where $\Omega$ is the $(1,2)$-tensor field on $M$ defined as

$$
\Omega(X, Y)=\left(\nabla_{X} \phi\right) Y-g(X+h X, Y) \xi+\eta(Y)(X+h X)
$$

for vector fields $X, Y$ on $M$ (see in [7] (Proposition 2.1)). Here, we remind that an almost contact Riemannian manifold is Sasakian manifold if and only if it satisfies

$$
\left(\nabla_{X} \phi\right) Y=g(X, Y) \xi-\eta(Y) X
$$

We also find that from (7) and (2) a Sasakian manifold is a K-contact manifold. Denote by $R$ the Riemannian curvature tensor defined by

$$
R(X, Y) Z=\nabla_{X}\left(\nabla_{Y} Z\right)-\nabla_{Y}\left(\nabla_{X} Z\right)-\nabla_{[X, Y]} Z
$$

for all vector fields $X, Y, Z$. Then, a contact Riemanian manifold is a Sasakian manifold if and only if it satisfies

$$
R(X, Y) \xi=\eta(Y) X-\eta(X) Y
$$

for all vector fields $X$ and $Y$ on the manifold.

Please refer to the works in [8-10] for more details about almost contact structures and their associated (almost) CR structures.

\section{A Characterization of the Unit Sphere}

In this section, in order to give a characterization of the unit sphere, we first give a definition.

Definition 3. Let $(M ; \eta, \xi, \phi, g)$ be an almost contact Riemannian manifold. Then, a regular curve $\gamma(s)$ whose tangent $\dot{\gamma}(s)$ belongs to $D_{\gamma(s)}$ along $\gamma(s)$ is called a transversal curve on $M$, where $s$ denotes the arc-length of $\gamma$.

In particular, when $M$ is a 3-dimensional contact manifold, $\gamma(s)$ is said to be a Legendre curve. Making a generalization of this, we defined a slant curve whose contact angle $\alpha(s)=$ $g(\dot{\gamma}(s), \xi)$ is constant along $\gamma[11]$.

Definition 4. Let $\gamma(s)$ be a transversal geodesic on an almost contact Riemannian manifold $(M ; \eta, \xi, \phi, g)$. If the contact distribution $D$ is invariant by the Jacobi operator $R_{\gamma}=R(\cdot, \dot{\gamma}) \dot{\gamma}$, that is, $R_{\gamma}(D) \subset D$, then we have that $R_{\gamma}: D \rightarrow D$ is a self-adjoint operator along $\gamma$. We call $R_{\gamma}$ the transversal Jacobi operator along $\gamma$. 
We prepare the following lemmas.

Lemma 1. Let $(M ; \eta, \xi, \phi, g)$ be a contact Riemannian manifold. For any $p \in M$ and any $v \in D_{p}$, let $\gamma(s)$ be a geodesic with $\dot{\gamma}(0)=v$ and $\gamma(0)=p$. Then, $\gamma(s)$ is a transversal curve if and only if $M$ is a K-contact manifold.

Proof. Differentiating $\eta(\gamma(s))$ covariantly along $\gamma(s)$, then we have

$$
g(\phi \dot{\gamma}(0)+\phi h \dot{\gamma}(0), \dot{\gamma}(0))=0,
$$

which yields that $g(\phi h v, v)=0$ for any $v \in D_{p}$ and $p \in M$. Since $\phi h$ is a self-adjoint operator, taking a polarization, then we have $g(\phi h v, w)=0$ for any $v, w \in D_{p}$ and $p \in M$. Therefore, we have $\phi h=0$, and then $h=0$. Conversely, if $(M ; \eta, \xi, \phi, g)$ is a $K$-contact manifold, we easily find that $\eta(\dot{\gamma}(s))$ is constant along $\gamma(s)$.

Lemma 2. Let $(M ; \eta, \xi, \phi, g)$ be a K-contact manifold. Then, $R_{\gamma}(D) \subset D$ for any transversal geodesic $\gamma(s)$ on $M$ if and only if the associated pseudo-Hermitian structure is CR-integrable.

Proof. As $(M ; \eta, \xi, \phi, g)$ is a $K$-contact manifold, we have

$$
R(X, Y) \xi=\left(\nabla_{Y} \phi\right) X-\left(\nabla_{X} \phi\right) Y
$$

for all vector fields $X, Y$ in $M$. (We can easily get this identity from (4) and the definition of $R$.) Suppose that the associated pseudo-Hermitian structure is CR-integrable. Then, we have

$$
g\left(\left(\nabla_{U} \phi\right) V, W\right)=0
$$

for any vector fields $U, V, W \in D$. Indeed, the condition (9) is equivalent to CR-integrability (cf. [12]). Then, from (8) and (9) we have

$$
\begin{aligned}
g(R(V, \dot{\gamma}) \dot{\gamma}, \tilde{\xi}) & =-g(R(V, \dot{\gamma}) \xi, \dot{\gamma}) \\
& =g\left(\left(\nabla_{V} \phi\right) \dot{\gamma}-\left(\nabla_{\dot{\gamma}} \phi\right) V \cdot \dot{\gamma}\right) \\
& =0
\end{aligned}
$$

for any vector fields $V \in D$, where $\gamma=\gamma(s)$ is a transversal geodesic. Therefore, we have $D$ is invariant by $R_{\gamma}$. Conversely, we assume that $R_{\gamma}(D) \subset D$ for any transversal geodesic $\gamma$. Then by using polarization we have $g(R(V, U) W, \xi)+g(R(V, W) U, \xi)=0$ for any vector fields $U, V, W \in D$. Use (8) to obtain

$$
g\left(\left(\nabla_{U} \phi\right) V-\left(\nabla_{V} \phi\right) U, W\right)+g\left(\left(\nabla_{W} \phi\right) V-\left(\nabla_{V} \phi\right) W, U\right)=0 .
$$

On the other hand, from the definition of the fundamental two form $\Phi$, it is immediate that $d \Phi=0$ or, equivalently,

$$
0=g\left(\left(\nabla_{X} \phi\right) Y, Z\right)+g\left(\left(\nabla_{Y} \phi\right) Z, X\right)+g\left(\left(\nabla_{Z} \phi\right) X, Y\right)
$$

for all vector fields $X, Y, Z$ on $M$. Using (12) in (11), then we have

$$
g\left(\left(\nabla_{W} \phi\right) U+\left(\nabla_{U} \phi\right) W, V\right)=0
$$

for any vector fields $U, V, W \in D$. As $g\left(\left(\nabla_{W} \phi\right) U, V\right)+g\left(\left(\nabla_{U} \phi\right) V, W\right)+g\left(\left(\nabla_{V} \phi\right) W, U\right)=0$, using (13) and $g\left(\left(\nabla_{X} \phi\right) Y, Z\right)=-g\left(\left(\nabla_{X} \phi\right) Z, Y\right)$, then we have $g\left(\left(\nabla_{W} \phi\right) U, V\right)=0$ for any vector fields $U, V, W \in D$. Thus, we have proved Lemma 2 .

Now, we prove the following. 
Theorem 1. Let $(M ; \eta, \xi, \phi, g)$ be a complete and simply connected K-contact manifold. Then, $M$ is isometric to the unit sphere if and only if the eigenvalues of the transversal Jacobi operator $R_{\gamma}$ are constant and their eigenspaces are parallel along any transversal geodesic $\gamma$.

Proof. Let $(M ; \eta, \xi, \phi, g)$ be a $K$-contact manifold and let $\gamma(s)$ be a transversal geodesic parametrized by the arc-length $s$ and $R_{\gamma}$ be the transversal Jacobi operator. Then, due to Lemma 2, we already know that $(M ; \eta, \xi, \phi, g)$ is CR-integrable, that is, it is a Sasakian manifold. Suppose that the eigenvalues of the transversal Jacobi operator $R_{\gamma}$ are constant and their eigenspaces are parallel along any transversal geodesic $\gamma$. Then, the self-adjoint operator $R_{\gamma}: D \rightarrow D$ is diagonalizable by parallel orthonormal frame fields along a transversal geodesic $\gamma$. We may write

$$
R_{\gamma} E_{i}=\lambda_{i} E_{i}, \quad i=1,2, \cdots, 2 n-2,
$$

where $\nabla_{\dot{\gamma}} E_{i}=0, E_{i}(\gamma(0))=e_{i} \perp \xi_{p}, \dot{\gamma}\left(\lambda_{i}\right)=0$. Differentiating (14) covariantly along $\gamma$, then we have $\left(\nabla_{\dot{\gamma}} R\right)\left(E_{i}, \dot{\gamma}\right) \dot{\gamma}=0$ for any transversal geodesic $\gamma$, where $i=1,2, \cdots, 2 n-2$. From this, we get $g\left(\left(\nabla_{\dot{\gamma}} R\right)(U, \dot{\gamma}) \dot{\gamma}, W\right)=0$ for any vector fields $U, W \in D$, and then, we have $g\left(\left(\nabla_{V} R\right)(U, V) V, W\right)=0$ for any $U, V, W \in D$. Using polarization, we have $g\left(\left(\nabla_{V_{1}} R\right)\left(U, V_{2}\right) V_{3}, W\right)=0$ for any $U, W, V_{1}, V_{2}, V_{3} \in D$, which means that $M$ is a locally $\phi$-symmetric space in the sense of Takahashi [13]. Now we compute

$$
\begin{aligned}
0 & =g\left(\left(\nabla_{\dot{\gamma}} R\right)(U, \dot{\gamma}) \dot{\gamma}, \xi\right) \\
& =g\left(\nabla_{\dot{\gamma}} R(U, \dot{\gamma}) \dot{\gamma}-R\left(\nabla_{\dot{\gamma}} U, \dot{\gamma}\right) \dot{\gamma}, \xi\right) \\
& =g(R(U, \dot{\gamma}) \dot{\gamma}, \phi \dot{\gamma})-g\left(R\left(\nabla_{\dot{\gamma}} U, \dot{\gamma}\right) \dot{\gamma}, \xi\right) \quad\left(\because R_{\gamma}(D) \subset D \text { and }(4)\right) \\
& =g(R(U, \dot{\gamma}) \dot{\gamma}, \phi \dot{\gamma})+g\left(R\left(\nabla_{\dot{\gamma}} U, \dot{\gamma}\right) \xi, \dot{\gamma}\right) \\
& =g(R(U, \dot{\gamma}) \dot{\gamma}, \phi \dot{\gamma})-\eta\left(\nabla_{\dot{\gamma}} U\right) \quad(\because(2)) \\
& =g(R(U, \dot{\gamma}) \dot{\gamma}, \phi \dot{\gamma})-g\left(\nabla_{\dot{\gamma}} U, \xi\right) \\
& =g(R(U, \dot{\gamma}) \dot{\gamma}, \phi \dot{\gamma})+g\left(U, \nabla_{\dot{\gamma}} \xi\right) \\
& =g(R(U, \dot{\gamma}) \dot{\gamma}, \phi \dot{\gamma})-g(U, \phi \dot{\gamma}) \quad(\because(4))
\end{aligned}
$$

for any vector field $U \in D$. Assuming $U=\phi \dot{\gamma}$, then we have $g(R(\dot{\gamma}, \phi \dot{\gamma}) \phi \dot{\gamma}, \dot{\gamma})=1$. This implies $g(R(V, \phi V) \phi V, V)=1$ for any unit vector field $V \in D$. We have that $M$ is a Sasakian space of constant $\phi$-sectional curvature 1 . Thus, we have that $M$ is locally isometric to the unit sphere. As $M$ is complete and simply connected, it is (globally) isometric to the unit sphere. In order to prove the converse, we assume that $M$ is isometric to a Sasakian unit sphere and that $R_{v} e_{i}=\lambda_{i} e_{i}, i=1,2, \cdots, 2 n-2$, where $v \in D_{p}$ and $\left\{e_{i}\right\}$ is an orthonormal basis of $D_{p} \subset T_{p} M$. Let $\gamma(s)$ be a geodesic with $\gamma(0)=p, \dot{\gamma}(0)=v$ and let $\left\{E_{i}\right\}$ be a parallel orthonormal frame field along $\gamma$ with $E_{i}(0)=e_{i}, i=1,2, \cdots, 2 n-2$. Differentiate covariantly $R_{\dot{\gamma}} E_{i}$ and $\lambda_{i} E_{i}$, respectively, along $\gamma$, then we find that both items are parallel along $\gamma$. In addition, as at the initial point $\gamma(0)=p$ they coincide, that is, $R_{v} e_{i}=\lambda_{i} e_{i}$ we have that $R_{\dot{\gamma}} E_{i}=\lambda_{i} E_{i}, i=1,2, \cdots, 2 n-2$. Therefore, we have completed the proof.

Corollary 1. Let $(M ; \eta, \xi, \phi, g)$ be a complete and simply connected K-contact manifold satisfying $R_{\gamma}(D) \subset D$ for any transversal geodesic $\gamma$. Then, $M$ is the Sasakian sphere if and only if it satisfies $R_{\gamma}^{\prime}(D) \subset D$ for any transversal geodesic $\gamma$, where $R_{\gamma}^{\prime}=\left(\nabla_{\dot{\gamma}} R\right)(\cdot, \dot{\gamma}) \dot{\gamma}$.

\section{Real Hypersurfaces of a Non-Flat Complex Space Form}

An oriented real hypersurface $M$ of a Kähler manifold $\left(\widetilde{M}_{n} ; \widetilde{J}, \widetilde{g}\right)$ of complex dimension $n$ admits an almost contact metric structure $(\eta, \xi, \phi, g)$ :

$$
\widetilde{J} X=\phi X+\eta(X) N, \quad \widetilde{J} N=-\xi
$$


for any vector field $X$ tangent to $M$ and the unit normal vector field $N$. Denote by $g$ the induced Riemannian metric on $M$. Then, $(\eta, \phi, \xi, g)$ satisfy (1) and (2). Recall the Gauss and Weingarten formulas for $M$ :

$$
\widetilde{\nabla}_{X} Y=\nabla_{X} Y+g(A X, Y) N, \widetilde{\nabla}_{X} N=-A X
$$

for any tangent vector fields $X, Y$, where $\widetilde{\nabla}$ and $\nabla$ denote the Levi-Civita connections of $\left(\widetilde{M}_{n}, \widetilde{g}\right)$ and $(M, g)$, respectively, and $A$ is the shape operator field. As $\widetilde{\nabla} J=0$, we obtain

$$
\begin{gathered}
\left(\nabla_{X} \phi\right) Y=\eta(Y) A X-g(A X, Y) \xi \\
\nabla_{X} \xi=\phi A X .
\end{gathered}
$$

If we put $\left.\phi\right|_{D}=J$, then the associated pseudo-Hermitian structure $(\eta, J)$ is CR-integrable. Indeed, using (16) and (17), it is seen that a real hypersurface of a Kähler manifold satisfies (5) and (6). An eigenvector corresponding to an eigenvalue of $A$ is called a principal (curvature) vector. From (17), it follows easily that:

Lemma 3. The Reeb vector field $\xi$ is principal if and only if its integral curves are geodesics.

A real hypersurface of a Kähler manifold is said to be a Hopf hypersurface if the Reeb vector $\xi$ is a principal curvature vector.

Assume that $\widetilde{M}_{n}(c), n \geq 2, c \neq 0$, is a complex space form of constant holomorphic sectional curvature $c$. Then, we have the following Gauss equation for a real hypersurface $M$ in $\widetilde{M}_{n}(c)$ :

$$
\begin{aligned}
R(X, Y) Z= & \frac{c}{4}\{g(Y, Z) X-g(X, Z) Y \\
& +g(\phi Y, Z) \phi X-g(\phi X, Z) \phi Y-2 g(\phi X, Y) \phi Z\} \\
& +g(A Y, Z) A X-g(A X, Z) A Y
\end{aligned}
$$

for any tangent vector fields $X, Y, Z$ on $M$. By making use of the six types homogeneous real hypersurfaces of $P_{n} \mathbb{C}$ given in [14], and the tube construction of a Hopf hypersurface realized in [15], M. Kimura proved the following (local) classification theorem.

Theorem 2 ([16]). Let $M$ be a Hopf hypersurface of $P_{n} \mathbb{C}$. Then, $M$ has constant principal curvatures if and only if $M$ is locally congruent to one of the following.

$\left(A_{1}\right)$ a geodesic hypersphere of radius $r$, where $0<r<\frac{\pi}{2}$,

$\left(A_{2}\right)$ a tube of radius $r$ over a totally geodesic $P_{l} \mathbb{C}(1 \leq l \leq n-2)$, where $0<r<\frac{\pi}{2}$,

(B) a tube of radius r over a complex quadric $Q^{n-1}$ and $P_{n} \mathbb{R}$, where $0<r<\frac{\pi}{4}$,

(C) a tube of radius $r$ over $P_{1} \mathbb{C} \times P_{\frac{n-1}{2}} \mathbb{C}$, where $0<r<\frac{\pi}{4}$ and $n(\geq 5)$ is odd,

(D) a tube of radius r over a complex Grassmann $G_{2,5} \mathbb{C}$, where $0<r<\frac{\pi}{4}$ and $n=9$,

$(E)$ a tube of radius r over a Hermitian symmetric space $S O(10) / U(5)$, where $0<r<\frac{\pi}{4}$ and $n=15$.

J. Berndt proved the following theorem for the case $H_{n} \mathbb{C}$.

Theorem 3 ([17]). Let $M$ be a Hopf hypersurface of $H_{n} \mathbb{C}$. Then, $M$ has constant principal curvatures if and only if $M$ is locally congruent to one of the following.

$\left(A_{0}\right)$ a horosphere,

$\left(A_{1}\right)$ a geodesic hypersphere or a tube over a complex hyperbolic hyperplane $H_{n-1} \mathbb{C}$,

$\left(A_{2}\right)$ a tube over a totally geodesic $H_{l} \mathbb{C}(1 \leq l \leq n-2)$,

(B) a tube over a totally real hyperbolic space $H_{n} \mathbb{R}$.

We call simply type $(A)$ for real hypersurfaces of type $A_{1}, A_{2}$ in $P_{n} \mathbb{C}$ and those of type $A_{0}, A_{1}$, or $A_{2}$ in $H_{n} \mathbb{C}$. 
Ruled real hypersurfaces are foliated real hypersurfaces with totally geodesic submanifolds of $P_{n} \mathbb{C}$ as leaves of codimension 1 [18]. Let $\bar{M}$ be a hypersurface in $S^{2 n+1}$ defined by

$$
\begin{gathered}
\left\{\left(r e^{i t} \cos \theta, r e^{i t} \sin \theta,\left(1-r^{2}\right)^{1 / 2} z_{2}, \cdots,\left(1-r^{2}\right)^{1 / 2} z_{n}\right) \in \mathbb{C}^{n+1} \mid\right. \\
\left.\sum_{j=2}^{n}\left|z_{j}\right|^{2}=1,0<r<1,0 \leq t, \theta<2 \pi\right\} .
\end{gathered}
$$

Then the Hopf image $M$ of $\bar{M}$ is a minimal ruled hypersurface in $P_{n} \mathbb{C}$. Indeed, the shape operator is given as follows, $A \xi=\frac{\sqrt{1-r^{2}}}{r} U, A U=\frac{\sqrt{1-r^{2}}}{r} \xi$ and $A Z=0$ for any $\mathrm{Z} \perp\{\xi, U\}$, where $U \perp \xi$ is unit vector field. In analogous way, a minimal ruled real hypersurface in $H_{n} \mathbb{C}$ provided in [19]. In general, the shape operator of ruled real hypersurfaces in $P_{n} \mathbb{C}$ or $H_{n} \mathbb{C}$ is written as follows,

$$
\begin{aligned}
& A \xi=\alpha \xi+\mu U(\mu \neq 0), \\
& A U=\mu \xi, \\
& A Z=0
\end{aligned}
$$

for any $Z \perp\{\xi, U\}$, where $U \perp \xi$ is unit vector field, $\alpha$ and $\mu$ are functions on $M$.

Theorem 4 ([18,19]). Let $M$ be a real hypersurface in a non-flat complex space form $\widetilde{M}$. Then $M$ is a ruled real hypersurface if and only if $g(A X, Y)=0$ for any tangent vectors $X, Y$ orthogonal to §on $M$.

Recently, a new description of ruled real hypersurfaces in $P_{n} \mathbb{C}$ as the image of a curve in $P_{n} \mathbb{C}$ under the polar map was given in [20]. Note that such a ruled real hypersurface has constant $\phi$-sectional curvature, but it is not complete. On the other hand, M. Kimura, $\mathrm{S}$. Maeda, and H. Tanabe [21] gave an explicit construction of ruled real hypersurfaces in $H_{n} \mathbb{C}$ from a real curve in the indefinite complex projective space $P_{n}^{1} \mathbb{C}$ of index 2 . Indeed, identifying all totally geodesic complex hyperplanes $H_{n-1} \mathbb{C}$ in $H_{n} \mathbb{C}$ with the indefinite complex projective space $P_{n}^{1} \mathbb{C}$ and making the correspondence a real 1-dimensional curve $\delta$ in $P_{n}^{1} \mathbb{C}$ to a ruled real hypersurface in $H_{n} \mathbb{C}$, they showed that the curve $\delta$ is a pregeodesic in $P_{n}^{1} \mathbb{C}$ if and only if the ruled real hypersurface $M$ is minimal. There are three types of minimal ruled real hypersurfaces: elliptic type, parabolic type, and axial type, which correspond to a spacelike, lightlike, and timelike pregeodesic in $P_{n}^{1} \mathbb{C}$, respectively. Among them, ruled real hypersurfaces of parabolic type and axial type are complete.

Now, we have the following.

Lemma 4. Let $M$ be a real hypersurface in a Kähler manifold. For any $p \in M$ and any $v \in D_{p}$, let $\gamma(s)$ be a geodesic with $\dot{\gamma}(0)=v$ and $\gamma(0)=p$. Then, $\gamma(s)$ is a transversal curve if and only if $M$ satisfies $(*) g((\phi A-A \phi) X, Y)=0$ for any vector fields $X, Y \perp \xi$.

Theorem 5. Let $M$ be a real hypersurface in a non-flat complex space form $\widetilde{M}_{n}(c), c \neq 0$. Suppose that $M$ satisfies the condition $(*)$. Then $R_{\gamma}(D) \subset D$ for any transversal geodesic $\gamma(s)$ on $M$ if and only if $M$ is locally congruent to either a real hypersurface of type $(A)$ or a ruled real hypersurface.

Proof. From Gauss equation (18), we have

$$
R_{\gamma}(X)=R(X, \dot{\gamma}) \dot{\gamma}=\frac{c}{4}\{X-g(X, \dot{\gamma}) \dot{\gamma}-3 g(\phi X, \dot{\gamma}) \phi \dot{\gamma}\}+g(A \dot{\gamma}, \dot{\gamma}) A X-g(A X, \dot{\gamma}) A \dot{\gamma}
$$

for any vector field $X \perp \xi$ and for any transversal geodesic $\gamma$ on $M$. From (20), we have that $R_{\gamma}(D) \subset D$ if and only if

$$
g(A V, V) g(A X, \xi)-g(A X, V) g(A V, \xi)=0
$$


for any vector field $V \perp \xi$ on $M$. Suppose that $M$ is a Hopf hypersurface and assume that $A \xi=\alpha \xi$. Then, we at once see that $M$ satisfies (21). Together with Lemma 4, we see that $M$ satisfies $A \phi=\phi A$. Due to Okumura's result [22] (in the case $P_{n} \mathbb{C}$ ) and Montiel and Romero's result [23] (in the case $H_{n} \mathbb{C}$ ), we have that $M$ is of type $(A)$ real hypersurfaces in $P_{n} \mathbb{C}$ or $H_{n} \mathbb{C}$. Now, we consider the case $M$ is non-Hopf. Then, we assume that $A \xi=\alpha \xi+\mu U$, where $\mu$ is a function in $M$ and $U$ is a unit vector field orthogonal to $\xi$. Plugging this into (21), then we have

$$
g(A V, V) g(X, U)=g(A X, V) g(U, V),
$$

where $\mu \neq 0$. If we put $V=\phi U$ and $X=U$ in (22), then we get $g(A \phi U, \phi U)=0$. However, as $A$ and $\phi$ commute on $D$, we have $g(A U, U)=0$. We put $V=U$ and $X=Z \in$ $\operatorname{Span}\{\xi, U\}^{\perp}$ in (22), then we get $g(A Z, U)=0$. Thus, we find that $M$ is locally congruent to a ruled real hypersurface.

Assume that $R_{\gamma}(D) \subset D$. Then, $R_{\gamma}: D \rightarrow D$ is a self-adjoint operator along $\gamma$. In a previous paper [24] (proof of Theorem 2), we had that a real hypersurface of type $(A)$ in $P_{n} \mathbb{C}$ or $H_{n} \mathbb{C}$ fails to satisfy $g\left(R_{\gamma}^{\prime} V, \xi\right)=0$ for any vector field $V \perp \xi$. As a ruled real hypersurface in complex projective space $P_{n} \mathbb{C}$ is not complete, then we have

Theorem 6. Let $M$ be a complete real hypersurface in a non-flat complex space form. Then, $M$ is congruent to a complete ruled real hypersurface in complex hyperbolic space $H_{n} \mathbb{C}$ if and only if the eigenvalues of the transversal Jacobi operator $R_{\gamma}$ are constant and their eigenspaces are parallel along any transversal geodesic $\gamma$.

Corollary 2. Let $M$ a complete real hypersurface in a non-flat complex space form satisfying $R_{\gamma}(D) \subset D$ for any transversal geodesic $\gamma$. Then, $M$ is a complete ruled real hypersurface if and only if it satisfies $R_{\gamma}^{\prime}(D) \subset D$ for any transversal geodesic $\gamma$, where $R_{\gamma}^{\prime}=\left(\nabla_{\dot{\gamma}} R\right)(\cdot, \dot{\gamma}) \dot{\gamma}$.

Author Contributions: Writing—original draft, J.T.C. and M.K. All authors have read and agreed to the published version of the manuscript.

Funding: J.T.C. was supported by Basic Science Research Program through the National Research Foundation of Korea (NRF) funded by the Ministry of Education, Science and Technology (2019R1F1A1040829). M. Kimura was supported by JSPS KAKENHI Grant Number JP20K03575.

Institutional Review Board Statement: Not applicable.

Informed Consent Statement: Not applicable.

Data Availability Statement: Data sharing not applicable.

Conflicts of Interest: The authors declare no conflicts of interest.

\section{References}

1. Sasaki, S. On differentiable manifolds with certain structures which are closely related to almost contact structure I. Tohoku Math. J. 1960, 12, 456-476. [CrossRef]

2. Webster, S.M. Pseudohermitian structures on a real hypersurface. J. Differ. Geom. 1978, 13, 25-41. [CrossRef]

3. Tanaka, N. On non-degenerate real hypersurfaces, graded Lie algebras and Cartan connections. Jpn. J. Math. 1976, 2, 131-190. [CrossRef]

4. Gray, J.W. Some global properties of contact structure. Ann. Math. 1959, 69, 421-450. [CrossRef]

5. Dragomir, S.; Tomassini, G. Differential Geometry and Analysis on CR Manifolds; Progress in Mathematics 246; Birkhäuser Boston, Inc.: Boston, MA, USA, 2006.

6. Sasaki, S.;Hatakeyama, Y. On differentiable manifolds with certain structures which are closely related to almost contact structure II. Tôhoku Math. J. 1961, 13, 281-294. [CrossRef]

7. Tanno, S. Variational problems on contact Riemannian manifolds. Trans. Am. Math. Soc. 1989, 22, 349-379. [CrossRef]

8. Blair, D.E. Riemannian Geometry of Contact and Symplectic Manifolds, 2nd ed.; Progress in Mathematics 203; Birkhäuser Boston, Inc.: Boston, MA, USA, 2010.

9. Cho, J.T. CR structures on real hypersurfaces of a complex space form. Publ. Math. Debrecen 1999, 54, $473-487$. 
10. Cho, J.T. Contact hypersurfaces and CR-symmetry. Ann. Mat. Pura Appl. 2020, 199, 1873-1884. [CrossRef]

11. Cho, J.T.;Inoguchi J.;Lee J. On slant curves in Sasakian 3-manifolds. Bull. Aust. Math. Soc. 2006, 74, 359-367. [CrossRef]

12. Boeckx, E.; Cho, J.T. $\eta$-parallel contact metric spaces. Differ. Geom. Appl. 2005, 22, 275-285. [CrossRef]

13. Takahashi, T. Sasakian $\phi$-symmetric spaces. Tôhoku Math. J. 1977, 29, 91-113. [CrossRef]

14. Takagi, R. On homogeneous real hypersurfaces in a complex projective space. Osaka J. Math. 1973, 19, $495-506$.

15. Cecil, T.E.; Ryan, P.J. Focal sets and real hypersurfaces in complex projective space. Trans. Amer. Math. Soc. 1982, 269, 481-499. [CrossRef]

16. Kimura, M. Real hypersurfaces and complex submanifolds in complex projective space. Trans. Amer. Math. Soc. 1986, 296, 137-149. [CrossRef]

17. Berndt, J. Real hypersurfaces with constant principal curvatures in complex hyperbolic space. J. Reine Angew. Math. 1989, 395, 132-141. [CrossRef]

18. Kimura, M. Sectional curvatures of holomorphic planes on a real hypersurfaces in $P^{n}(\mathbb{C})$. Math. Ann. 1987, $276,487-497$. [CrossRef]

19. Ahn, S.S.; Lee, S.-B.; Suh, Y.J. On ruled real hypersurfaces in a complex space form. Tsukuba J. Math. 1993, 17, 311-322. [CrossRef]

20. Cho, J.T.; Kimura, M. Real hypersurfaces with constant $\phi$-sectional curvature in complex projective space. Differ. Geom. Appl. 2020, 68, 101573. [CrossRef]

21. Kimura, M.; Maeda, S.; Tanabe, H. New construction of ruled real hypersurfaces in a complex hyperbolic space and its applications. Geom. Dedicata 2019. [CrossRef]

22. Okumura, M. On some real hypersurfaces of a complex projective space. Trans. Amer. Math. Soc. 1975, 212, 355-364. [CrossRef]

23. Montiel S.; Romero, A. On some real hypersurfaces of a complex hyperbolic space. Geom. Dedicata 1986, 20, 245-261. [CrossRef]

24. Cho, J.T.; Kimura, M. Tranversal symmetries on real hypersurfaces in a complex space form. Hiroshima Math. J. 2013, 43, 223-238. [CrossRef] 